\title{
Measurements of electron beam deflection and rf breakdown rate from a surface wave guided in metallic mm-wave accelerating structures
}

\author{
Massimo Dal Forno, ${ }^{1, *}$ Valery Dolgashev, ${ }^{1}$ Gordon Bowden, ${ }^{1}$ Christine Clarke, ${ }^{1}$ Mark Hogan, ${ }^{1}$ \\ Doug McCormick, ${ }^{1}$ Alexander Novokhatski, ${ }^{1}$ Brendan O'Shea, ${ }^{1}$ Bruno Spataro, ${ }^{2}$ \\ Stephen Weathersby, ${ }^{1}$ and Sami G. Tantawi ${ }^{1}$ \\ ${ }^{1}$ SLAC National Accelerator Laboratory, Menlo Park, California 94025, USA \\ ${ }^{2}$ INFN/LNF Frascati, Via E. Fermi, 40, Frascati (Roma) 00044, Italy
}

(Received 4 March 2018; published 13 September 2018)

\begin{abstract}
Experiments with high gradient traveling-wave mm-wave metallic accelerating structures were performed to research the physics of vacuum rf breakdowns. The accelerating structures are open, composed of two identical halves separated by an adjustable gap. The electromagnetic fields were induced by an ultrarelativistic electron beam at the Facility for Advanced Accelerator Experimental Tests (FACET) at SLAC National Accelerator Laboratory. These accelerating structures have a fundamental beamsynchronous mode with a frequency that varies from 95 to $140 \mathrm{GHz}$ depending on the gap width. When the gap is opened by more than half of a freespace wavelength, the corresponding synchronous mode remains trapped demonstrating behavior consistent with the so called "surface wave." This regime is of potential interest for femtosecond electron beam diagnostics and beam manipulation techniques. The behavior of the surface wave was characterized and the measured synchronous frequency, pulse length and beam deflection voltage are found to be in good agreement with simulations. The wave magnitude was changed by positioning the beam at different distances from the surface of a structure-half and the corresponding if breakdown rate was recorded. The breakdown rate was $4 \times 10^{-3}$ per pulse at a peak surface electric field of $0.3 \mathrm{GV} / \mathrm{m}$ and a pulse length of $60 \mathrm{ps}$.
\end{abstract}

DOI: 10.1103/PhysRevAccelBeams.21.091301

\section{INTRODUCTION}

As part of research on the physics of vacuum rf breakdowns we performed experiments with open mm-wave metallic accelerating structures, made with hard copper, hard copper-silver alloy and stainless steel. We reported the result of our previous experiments in [1-4]. The field in the structures were excited by an ultrarelativistic electron beam. The beam was generated by FACET [5], housed in the first $2 \mathrm{~km}$ of the SLAC linear accelerator [6]. The beam energy was $20.35 \mathrm{GeV}$. In the last experiment [4] we analyzed a travelling-wave accelerating structure, made of hard copper-silver alloy.

We changed the interaction with the beam by changing the gap width. By opening the gap, the number of trapped modes is reduced. We observed that a travelling-wave mode is trapped between the structures halves with oversized gaps, larger than a half wavelength. At a

\footnotetext{
*dalforno@slac.stanford.edu
}

Published by the American Physical Society under the terms of the Creative Commons Attribution 4.0 International license. Further distribution of this work must maintain attribution to the author(s) and the published article's title, journal citation, and DOI. gap of $7.5 \mathrm{~mm}$, the frequency of the trapped mode was $95 \mathrm{GHz}$. This behavior is consistent with the so called "surface wave," where the rf power is guided by the corrugations with no "radiative losses." The term "radiative losses" refers to rf power that is emitted out of the structure.

Surface waves have a long history. They were studied by C. Cutler for applications to communications during the second world war [7]. Cutler showed that guided waves do not necessarily need to be confined within physical boundaries. G. Goubau [8-10] presented single conductor surface wave transmission lines. W. Rotman studied a single surface corrugated waveguide [11]. A review of surface waves is presented by G. John [12]. H. M. Barlow [13] discussed the different forms of surface waves, behavior and applications. Similar effect was also studied by Smith-Purcell [14] and used to make high power $\mathrm{THz}$ sources [15].

In our experiment the surface wave is trapped between the structures halves with oversized gap in between. While the surface wave has resistive wall losses, it has no radiative losses. We experimentally characterized the beam-surface wave interaction with several measurements: by measuring radiated rf energy with a pyro-detector, by measuring the $\mathrm{rf}$ spectrum and the rf pulse length of the surface wave with a $\mathrm{mm}$-wave interferometer. 
TABLE I. Symbols and abbreviations used in this papers.

\begin{tabular}{ll}
\hline \hline$c$ & Speed of light \\
$a$ & Half gap of open structure or beampipe radius \\
$\lambda$ & Surface wave wavelength \\
$\kappa_{z}$ & Loss factor per unit length \\
$\kappa_{y}$ & Kick factor per unit length (of the vertical deflection) \\
$v_{g}$ & Group velocity \\
$\tau_{F}$ & Pulse length (filling time) \\
$\tau_{D}$ & Decay time (in power) \\
$E_{\text {acc }}$ & Accelerating gradient \\
$\Delta \phi$ & Phase advance per cell \\
$R$ & Shunt impedance per unit length \\
$P$ & Power flow through the cavity cross-section \\
$Q_{0}$ & Unloaded quality factor \\
$q$ & Bunch charge \\
$\sigma_{z}$ & r.m.s. bunch length \\
$L$ & Active length of the structure \\
$e$ & Electron charge \\
$u$ & Relative horizontal coordinate system \\
$v$ & Relative vertical coordinate system \\
$\Delta v$ & Beam-structure distance \\
$\Delta y$ & Vertical beam displacement on the diagnostic screen \\
$\theta_{y}$ & Vertical kick angle \\
$V_{y}$ & Vertical deflecting voltage \\
$E$ & Beam energy \\
$R_{34}$ & Beam optics coefficient that converts a beam \\
\hline \hline
\end{tabular}

We reported in the previous papers [1-4] the rf breakdown rate measurements performed with small gaps and with the electron beam travelling along the central axis between the two structure halves. This regime corresponds to the typical operation of particle accelerators. This paper will present the data obtained in a different regime, where the beam axis has a significant offset with respect the central axis of the structure. This mode of operation generates transverse fields which could be used for beam deflection and similar beam manipulation. In these experiments we used our structure at large gaps, and with the beam close to one of the structure halves, interacting with the surface wave.

We dedicated two shifts for the study of surface waves, on November 21 and November 30, 2015. The results are presented in the experimental section of this paper.

The previous papers [2-4] present the measurements of beam deflection in a plane parallel to the structure surface. This paper presents the measurements of beam deflection towards the surface of the structure, with different beamstructure half distances.

This paper is divided into four sections. Section I is the introduction. In Sec. II we present the geometry of the structure and rf simulations of the surface-wave. Section III presents the experimental setup. Section IV presents the experimental results, the measurements of pulse length, beam deflection and the breakdown rate measurements of a surface wave.

All symbols used in this paper are presented in Table I.

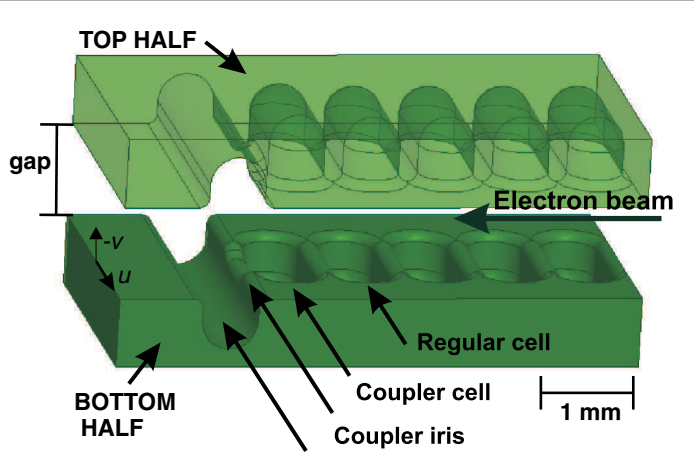

(a)

Output waveguide

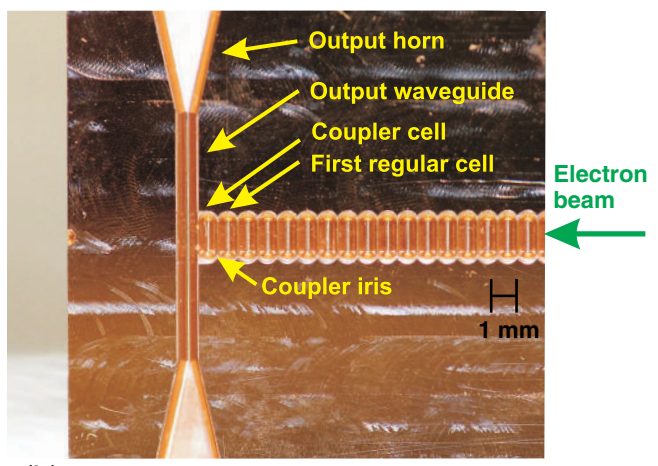

(b)

FIG. 1. Solid model of the output section of the $100 \mathrm{GHz}$ traveling wave accelerating structure, with the " $u, v$ " relative coordinate system used in this paper (a), and picture of the output section of the copper-silver structure (b). The gap between the structure halves can be changed with a motor.

\section{GEOMETRY AND RF PARAMETERS OF THE ACCELERATING STRUCTURE}

We conducted experiments with an open traveling wave structure made of hard copper-silver alloys [4]. The coppersilver alloy contains $0.085 \%$ silver. Since we do not have coherent MW-range mm-wave rf sources, the structure is electron-beam excited. It is composed of two separate

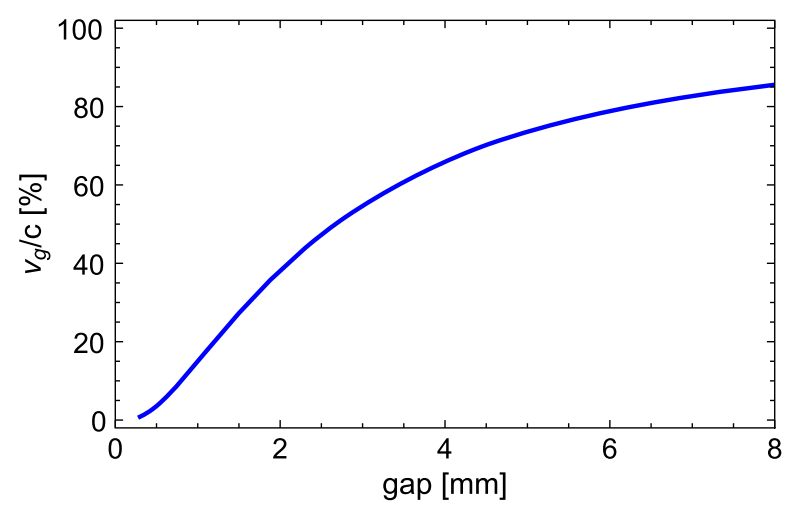

FIG. 2. Plot of group velocity of the fundamental mode, synchronous with the ultrarelativistic electron beam, as a function of the gap size. Simulations performed with Ansys HFSS for the fundamental mode. Details and formulas used to calculate the rf parameters are described in $[1,4]$. 
TABLE II. RF parameters of the regular cell of the $100 \mathrm{GHz}$ structure, for the fundamental mode, excited by $1 \mathrm{nC}$ electron bunch with $\sigma_{z}$ of $25 \mu \mathrm{m}$, traveling along the symmetry axis of the structure, calculated by using Ansys HFSS.

\begin{tabular}{lccccccccccc}
\hline \hline $\begin{array}{l}\mathrm{Gap} \\
{[\mathrm{mm}]}\end{array}$ & $\begin{array}{c}f \\
{[\mathrm{GHz}]}\end{array}$ & $\begin{array}{c}\Delta \phi \\
{[\mathrm{deg}]}\end{array}$ & $Q_{0}$ & $\begin{array}{c}R \\
{[M \Omega / \mathrm{m}]}\end{array}$ & $\begin{array}{c}\kappa_{z} \\
{[\mathrm{MV} /(\mathrm{nC})]}\end{array}$ & $\begin{array}{c}v_{g} / c \\
{[\%]}\end{array}$ & $\begin{array}{c}l_{\text {att }} \\
{[\mathrm{mm}]}\end{array}$ & $\begin{array}{c}P \\
{[\mathrm{MW}]}\end{array}$ & $\begin{array}{c}E_{\mathrm{acc}} \\
{[\mathrm{MV} / \mathrm{m}]}\end{array}$ & $\begin{array}{c}E_{\max } \\
{[\mathrm{GV} / \mathrm{m}]}\end{array}$ & $\begin{array}{c}H_{\max } \\
{[\mathrm{MA} / \mathrm{m}]}\end{array}$ \\
\hline 0.3 & 136.27 & 130.81 & 2054 & 398 & 41.6 & 0.85 & 6.08145 & 0.108 & 84 & 0.200 & 0.364 \\
0.5 & 130.30 & 125.20 & 2021 & 298 & 31.15 & 3.54 & 25.4071 & 0.347 & 63 & 0.175 & 0.336 \\
0.7 & 126.01 & 121.07 & 2022 & 222 & 23.5 & 7.65 & 54.1256 & 0.588 & 47 & 0.155 & 0.301 \\
0.9 & 122.66 & 117.88 & 2046 & 166 & 17.87 & 12.42 & 86.8502 & 0.768 & 36 & 0.139 & 0.269 \\
1.1 & 119.93 & 115.24 & 2089 & 128 & 13.89 & 17.48 & 119.878 & 0.890 & 28 & 0.127 \\
1.3 & 117.59 & 113.04 & 2145 & 99 & 10.97 & 22.44 & 151.625 & 0.956 & 22 & 0.116 & 0.250 \\
1.5 & 115.70 & 111.12 & 2211 & 78.2 & 8.83 & 27.29 & 180.984 & 0.991 & 18 & 0.107 & 0.209 \\
1.7 & 113.90 & 109.43 & 2286 & 62.5 & 7.18 & 31.85 & 207.87 & 1.003 & 14 & 0.099 & 0.195 \\
1.9 & 112.34 & 107.92 & 2367 & 50.7 & 5.92 & 36.15 & 232.068 & 1.002 & 12 & 0.091 & 0.179 \\
2 & 111.60 & 107.24 & 2411 & 45.9 & 5.40 & 38.18 & 243.222 & 0.997 & 11 & 0.088 & 0.169 \\
4 & 102.30 & 98.31 & 3518 & 10.3 & 1.38 & 65.91 & 368.595 & 0.797 & 2.75 & 0.048 & 0.098 \\
6 & 97.46 & 93.63 & 4870 & 3.92 & 0.58 & 78.8 & 398.211 & 0.647 & 1.16 & 0.032 & 0.067 \\
7.5 & 95.00 & 91.25 & 5969 & 2.27 & 0.36 & 84.2 & 398.76 & 0.573 & 0.72 & 0.025 & 0.055 \\
\hline \hline
\end{tabular}

movable metal halves, where the cavities and couplers are milled into the flat side of metal blocks. All the edges have been rounded to minimize the peak fields. The whole structure is $10 \mathrm{~cm}$ long, with $\mathrm{L}=8.96 \mathrm{~cm}$ active length of the accelerating cavities. Figure 1(a) shows a solid model of the output section of the $100 \mathrm{GHz}$ travelling wave accelerating structure. Figure 1(b) shows a photo of the output section of the copper-silver structure. More details of the geometry of the accelerating structure could be found in papers $[1,4]$.

The fields were excited by the FACET ultrarelativistic electron beam. To characterize the beam-structure interaction we simulated the periodic accelerating structure by using one regular cell. We calculate the rf parameters, accelerating gradients and peak fields by using the $\mathrm{rf}$ simulation code Ansys HFSS [16]. We used a modal expansion method, all the details and formulas to calculate the rf parameters are described in $[1,4]$. The rf parameters of the fundamental accelerating mode are listed in Table II, calculated with the gap varying from 0.3 to $7.5 \mathrm{~mm}$ and beam travelling on the axis of the structure. The number of high order modes interacting with the beam depends on the gap (see Fig. 11 of paper [1]). By opening the gap, the number of trapped modes is reduced. When the gap is opened beyond $1.3 \mathrm{~mm}$ only one mode remains trapped. With further opening the gap, the parameters of the wave such as frequency and group velocity are less dependent on the gap width. The plot of the group velocity of this fundamental mode is shown in Fig. 2 as a function of the gap size.

Typically the beampipe diameter of the accelerating structures is much smaller than the wavelength of the operating mode. For example at X-band $a / \lambda$ is around $0.1-$ 0.2 [17]. In our open accelerating structure we calculated

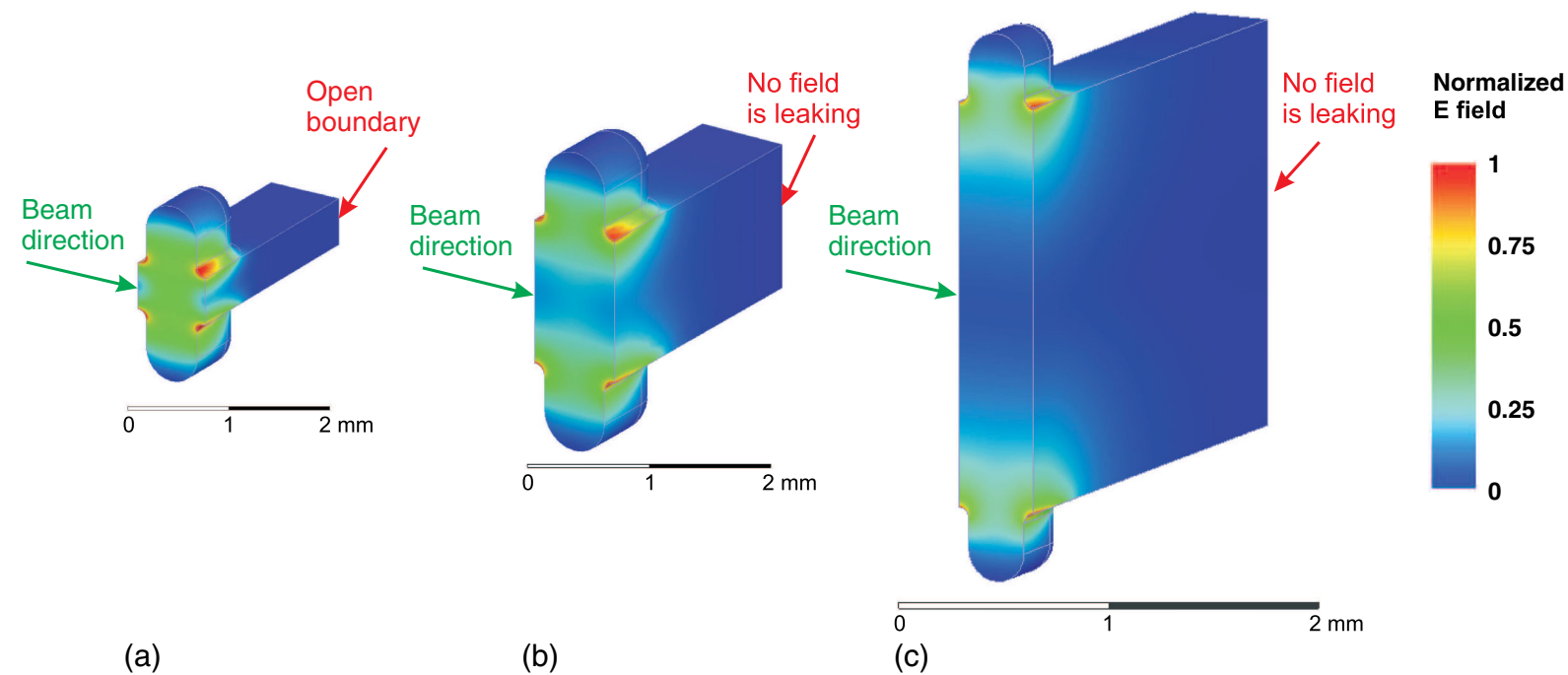

FIG. 3. Simulation of the synchronous wave in one period of the accelerating structure (half accelerating structure is shown): gap $=0.5 \mathrm{~mm}, \quad a / \lambda=0.108(f=130 \mathrm{GHz}) \quad(\mathrm{a}) ;$ gap $=1.3 \mathrm{~mm}, a / \lambda=0.26(f=118 \mathrm{GHz}) \quad(\mathrm{b}) ; \quad$ gap $=4 \mathrm{~mm}, \quad a / \lambda=0.7$ $(f=102 \mathrm{GHz})(\mathrm{c})$; in all three cases the mode is trapped. Simulations performed with Ansys HFSS for the fundamental mode. 


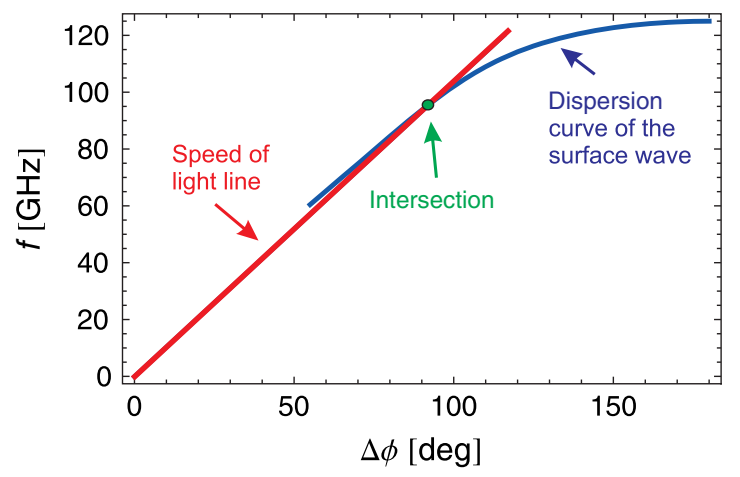

FIG. 4. Dispersion curve of the surface wave (blue curve) calculated with gap $=7.5 \mathrm{~mm}$, and speed of light (red line). The synchronous frequency is given by the intersection of the two curves. We did not plot the dispersion curve below 50 degrees of phase advance because below 50 degrees the mode is not trapped. Simulations performed with Ansys HFSS for the fundamental mode.

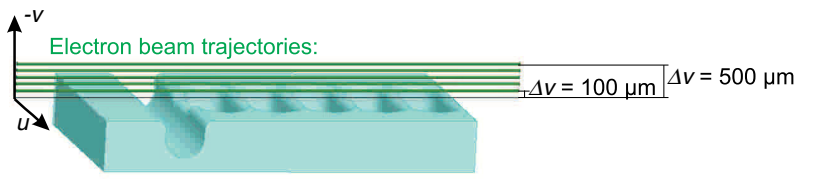

FIG. 5. Vertical relative position of the beam with respect to one half of the structure. the rf parameters for different gaps starting from $0.2 \mathrm{~mm}$, corresponding to $a / \lambda=0.05$. Figure 3 shows the simulation of the synchronous wave in one period of the accelerating structure: gap $=0.5 \mathrm{~mm}, a / \lambda=0.108, f=130 \mathrm{GHz}$ (a); gap $=1.3 \mathrm{~mm}, a / \lambda=0.26, f=118 \mathrm{GHz}(\mathrm{b}) ; \mathrm{gap}=4 \mathrm{~mm}$, $a / \lambda=0.7, f=102 \mathrm{GHz}$ (c). In all three cases the mode is trapped. Even when the gap is larger than $1.3 \mathrm{~mm}$, and the mode is above the beampipe cut-off, the rf power continues to be guided by the corrugations without radiative losses. This behavior is consistent with the so called "surface wave." On Fig. 4 we show the dispersion curve of the surface wave calculated with gap $=7.5 \mathrm{~mm}$. The intersection of the dispersion curve and the speed of light line (i.e., speed of the electron beam) gives the beam-synchronous frequency and the beam-synchronous phase advance per cell. In this case the group velocity is $84 \%$ of $\mathrm{c}$.

To understand the interaction between the electron beam and the surface wave, we calculated the loss factor with different distances from the structure half, as shown in Fig. 5 for gap $=6 \mathrm{~mm}$. To analyze the surface wave field profile, we created a mapping of the surface wave loss factor as a function of the $(u, v)$ beam position, by using Ansys HFSS. $(u, v)$ is a relative coordinate system, representing the beam position relative to the metal surface, indicated in Fig. 6(a-b). Figure 6(a) shows the solid model of one half of the accelerating structure, Fig. 6(b) shows the

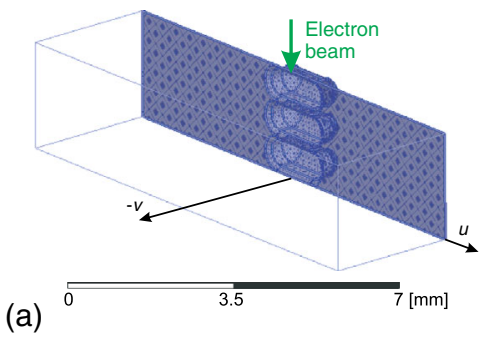

(b)

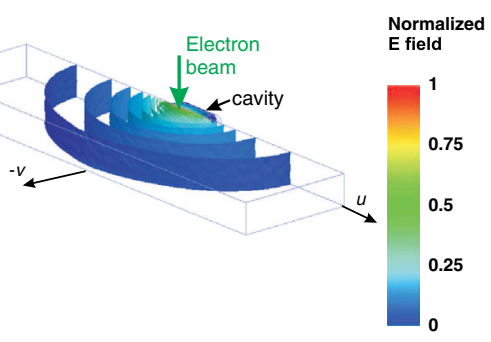

(c)

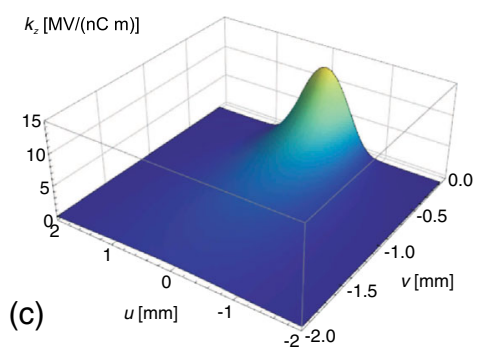

FIG. 6. (a) solid model of one half of the accelerating structure, (b) normalized electric field profile of the surface wave excited by the electron beam travelling $100 \mu \mathrm{m}$ far from the metal edge, (c) loss factor 3D plot as a function of the (u, $v$ ) beam position. (b) and (c) are calculated using $25 \mu \mathrm{m}$ of bunch length, and gap $=6 \mathrm{~mm}$, by using Ansys HFSS.
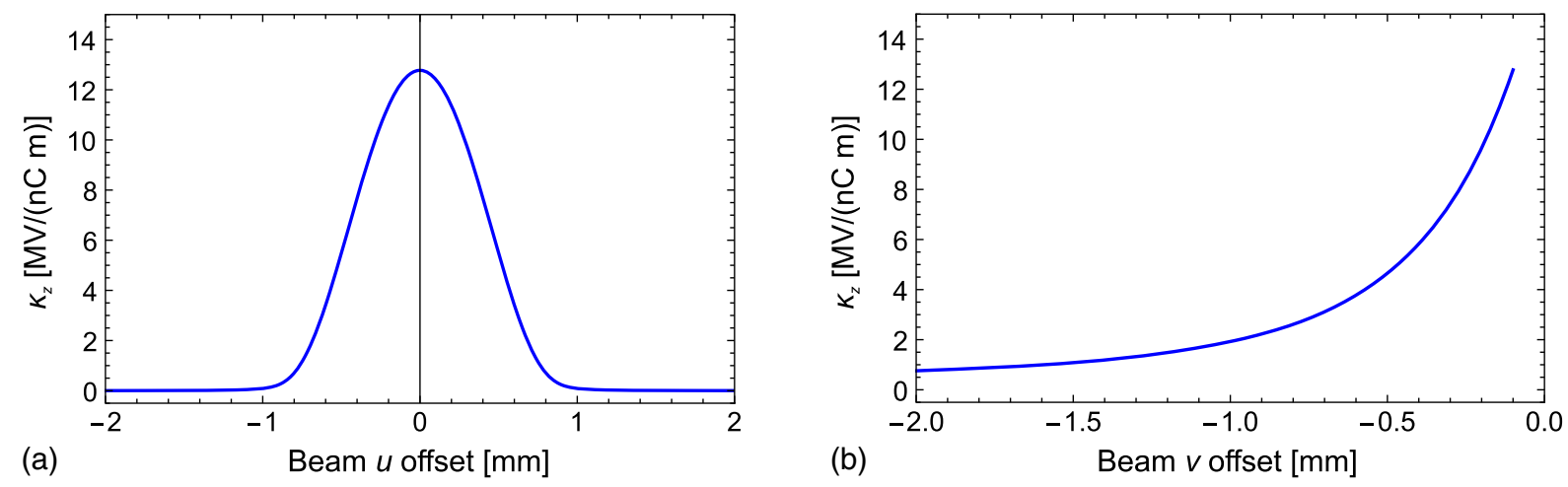

FIG. 7. Plot of the loss factor of the surface wave, (a) as a function of the $u$ position (with $v=-100 \mu \mathrm{m}$ ), (b) as a function of the $v$ position (with $u=0$ ), calculated with $\sigma_{z}=25 \mu \mathrm{m}$ and gap $=6 \mathrm{~mm}$, by using Ansys HFSS. 
electric field of the surface wave excited by the electron beam travelling $100 \mu \mathrm{m}$ far from the metal surface and Fig. 6(c) shows the 3D plot of the loss factor as a function of the $(u, v)$ beam position, with $25 \mu \mathrm{m}$ bunch length, with a gap of $6 \mathrm{~mm}$. This HFSS analysis considers only one resonant mode. The plot of the loss factor of the surface wave, as a function of the $u$ position (with $v=-100 \mu \mathrm{m}$ ), and as a function of the $v$ position (with $u=0$ ) are shown in Fig. 7, for a beam with $\sigma_{z}=25 \mu \mathrm{m}$.

\section{INSTALLATION OF THE ACCELERATING STRUCTURE}

The structure was manufactured by the company EDM Department Inc. [18], with precision milling, and cleaned according to SLAC procedure developed for the highgradient X-band program $[19,20]$. The structures, and the remote controlled stages used to align the structures to the beam, were housed in a vacuum chamber in the FACET experimental area, shown in Fig. 8. We assembled the two halves of the accelerating structure on remotely controlled motorized stages. Motorized stages are used to shift the structure horizontally $(\mathrm{X})$ or vertically $(\mathrm{Y})$ with respect to the electron beam. There was also a motor to adjust the gap between the two halves of the structure, shown in Fig. 9(b). A mirror is glued to the structure for laser alignment, and a phosphor screen is bolted to the edge of the structure to image the electron beam. The assembly is then installed in the experimental vacuum chamber. The structure antenna horns radiate the rf power towards pyrodetectors that sense the pulse energy and towards an interferometer that measures the frequency spectrum and the rf pulse length of the emitted radiation. For each experiment we recorded the pyrodetector voltage, which is proportional to the pulse energy emitted from the output waveguide horn.

We invented a new diagnostic tool able to reliably detect breakdowns [1]. Since breakdowns generate electron and ion currents, we incorporated an arc-detector unique to open structures. The two metal halves that compose the structure were electrically insulated from ground and each other using plastic film. We measured the field emission current and the breakdown current by measuring the voltage induced

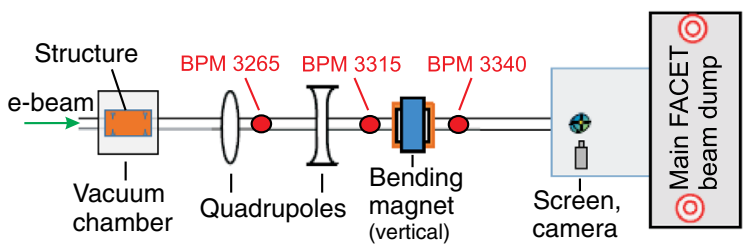

FIG. 8. Schematic of the experimental FACET region, where the structure is placed in the vacuum chamber with the horizontal, vertical and gap movers, the relevant beam position monitor (BPM) No. 3315, vertical bending magnet and the electron beam screen.
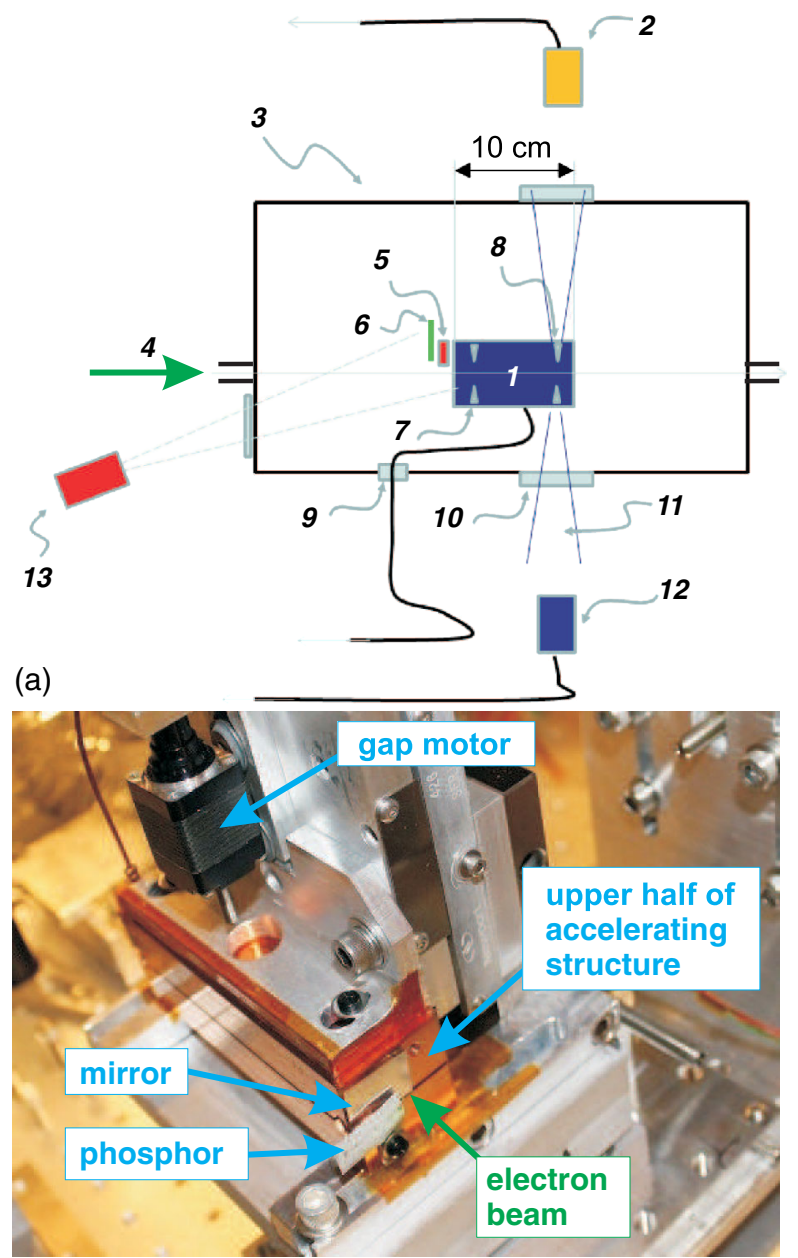

(b)

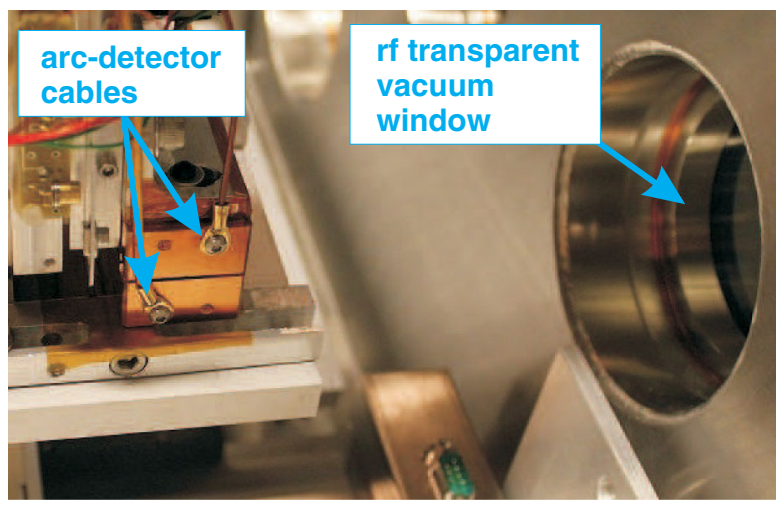

(c)

FIG. 9. Schematic of the experimental setup (a): accelerating structure (1), single-shot interferometer (2), vacuum chamber (3), electron beam (4), laser alignment mirror (5), phosphor screen (6), right reflected $\mathrm{rf}$ horn (7), left forward $\mathrm{rf}$ horn (8), vacuum feed-through (9), rf window (10), output rf beam (11), interferometer (12), video camera for beam-structure alignment (13). Accelerating structure assembled on remotely controlled motorized stages (b), back side of the structure with the arc-detector cables connected, installed in the vacuum chamber (c). 
TABLE III. Summary of the beam parameters of the two shifts, with date and time of each measurement. The beam energy is $20.35 \mathrm{GeV}$.

\begin{tabular}{lccccc}
\hline \hline & $\sigma_{x}[\mu \mathrm{m}]$ & $\sigma_{y}[\mu \mathrm{m}]$ & $\sigma_{z}[\mu \mathrm{m}]$ & $q[\mathrm{nC}]$ & gap $[\mathrm{mm}]$ \\
\hline Nov 21, 2015 & $25 \pm 6$ & $30 \pm 2$ & $25 \pm 2$ & 3.2 & 6 \\
& $\mathrm{~h} 14: 18$ & $\mathrm{~h} 14: 19$ & $\mathrm{~h} 14: 29$ & & \\
Nov 30, 2015 & $31 \pm 2$ & $20 \pm 6$ & $25 \pm 2$ & 2.7 & 7.5 \\
& $\mathrm{~h} 17: 30$ & $\mathrm{~h} 17: 27$ & $\mathrm{~h} 15: 28$ & & \\
\hline \hline
\end{tabular}

between the two separate metal blocks. This was achieved by connecting the two metal halves to an oscilloscope. More details on the breakdown rate measurements are reported in the breakdown rate measurement chapter.

After the installation, the vacuum chamber is closed and evacuated.

\section{EXPERIMENTAL RESULTS}

We dedicated two shifts to characterize the surface-wave. The measured parameter of the electron beam used in the experiment are shown in Table III. The transverse (horizontal and vertical) beam size was determined by using the wire scanner, while the bunch length was determined by using a transverse deflecting cavity [21,22]. We characterized the electron beam-surface wave interaction by several methods: measurement of the frequency and of the rf pulse length with an interferometer, measurement of the radiated rf energy with the pyrodetector, measurement of the beam deflection by using the beam position monitors and profile monitor and the measurement of the breakdown rate.

\section{A. Experimental procedure}

The experimental procedure is described by the following: (i) Detect the beam on the phosphor screen: if the electron beam hits the structure, it will damage delicate parts of the corrugations. Therefore, as a first step, we must steer the beam onto the phosphor screen, which is installed far from the corrugations. (ii) We used the following procedure to determine the midplane between the halves. For a fixed gap, we moved the structure vertically, up and down, until the beam halo is slightly intercepted by the metal, generating Bremsstrahlung radiation. The radiation was detected by photomultiplier tubes. We marked the positions of the motor where the peak photomultiplier voltages are approximately the same for the up and down positions. We took the average between these two motor positions to determine vertical center of the structure. By knowing the gap value $(2 a)$ and the central beam position, we shifted the structure (up or down) with the vertical mover of the amount " $a-\Delta v$ ", where " $\Delta v$ " is the desired beamstructure distance. Accuracy of these measurements are determined by the shape of the transverse distribution of the beam and resolution of mover positioning. The resolution of the mover motor is about a micrometer while bunch vertical size is $\sigma_{y}$. Since we cannot measure the exact transverse distribution of the beam, we conjecture that the upper bound on accuracy of the location of the vertical center of the structure is $\sigma_{y}$. (iii) Horizontal scan: once we placed the electron beam at the desired beam-structure distance, the structure is moved horizontally, allowing the beam to interact with the corrugations, generating electromagnetic fields. During each scan, we observed the magnitude of the energy pulses produced by the structure with pyrodetectors. (iv) The scan procedure is then repeated at different beamstructure distances. (v) Breakdown rate measurement: the beam is placed in the central axis. Breakdowns are counted using the arc-detector, while the frequency and pulse length are measured with the interferometer.

\section{B. Timeline of experiments}

We report the full timeline of the surface wave experiments in Fig. 10. We followed the format established for X-band rf breakdown experiments, showing the full history of the exposure of the structure to rf fields, and the corresponding accumulated rf breakdowns. When the beam moves close to either the top or bottom half of the structure the pyrodetector signal increases. During these shifts, we performed horizontal scans of the beam over the cavities (bell shaped curves of the pyrodetector signal), frequency and pulse length measurements and collection of breakdown statistics (corresponding to time intervals where the pyrodetector voltage is flat).

\section{Measurement of pulse length}

The method of measuring the pulse length of the emitted radiation is presented in paper [4]. We determine the $\mathrm{rf}$ pulse length of the surface wave with an interferometer. The results are shown in Fig. 11. They are in good agreement with the simulations (The definitions and calculations of the pulse lengths $\tau, \tau_{F}, \tau_{D}$ are discussed in Sec. IV of paper [4]). The measurements of pulse length contain statistical noise, contributing to the large error bar at gap $=3 \mathrm{~mm}$.

\section{Measurement of the beam deflection}

When the electron beam trajectory moves horizontally off-axis, its charge excites deflecting fields. Our previous papers [2-4] present the measurements of beam deflection in a plane parallel to the structure surface. This paper presents the measurements of beam deflection towards the surface of the structure, with different beam structure-half distances. We measured the vertical deflection (according to the orientation of Figs. 1, 5), when the beam is horizontally centered above the structure cells. We varied the vertical distance " $\Delta v$ ", as shown in Fig. 5 . The beam deflection is measured with the beam-position monitor No. 3315 (see Fig. 8), located after the structure. The beam optics between the test structure and the BPM No. 3315 converts the vertical kick angle $\theta_{y}$, generated by the structure, to a vertical beam displacement $\Delta y$, given by: 

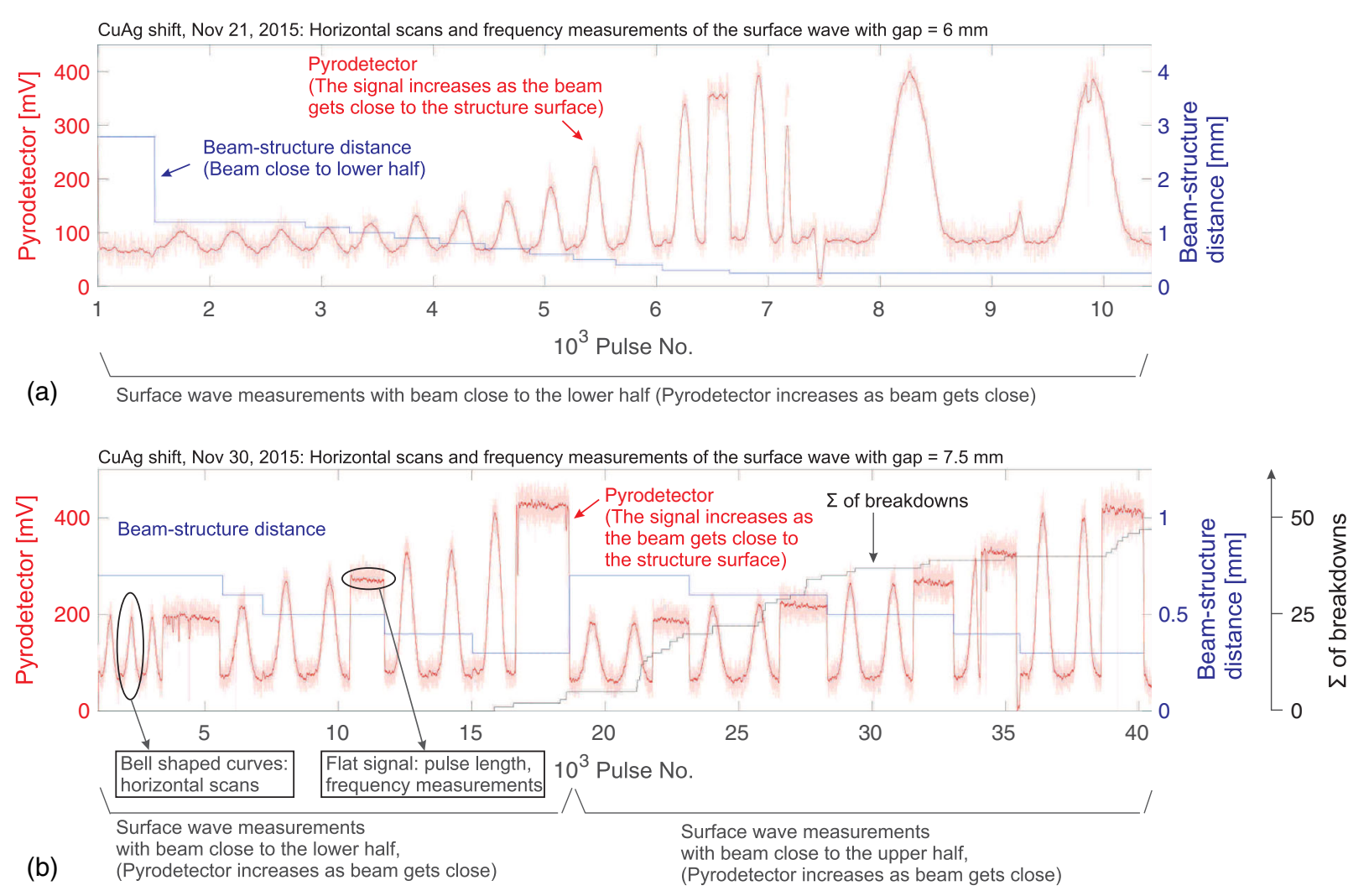

FIG. 10. Timeline of the surface wave measurements performed with the $100 \mathrm{GHz}$ copper-silver accelerating structure, shift of Nov 21, 2015 with $3.2 \mathrm{nC}$ of bunch charge, $\sigma_{z}=25 \mu \mathrm{m}$, the gap was $6 \mathrm{~mm}$ (a), shift of Nov 30, 2015 with $2.7 \mathrm{nC}$ of bunch charge, $\sigma_{z}=25 \mu \mathrm{m}$, the gap was $7.5 \mathrm{~mm}$ (b). The red plot is the reference pyrodetector signal, the blue is the beam-structure distance, the black is the integrated number of breakdowns, recorded with the arc-detector. Bell shaped curves of the pyrodetector signal are generated during the horizontal scans of the beam over the cavities and flat parts are the time intervals when we performed frequency and pulse length measurements and collection of breakdowns.

$$
\Delta y=R_{34} \cdot \theta_{y}=R_{34} \cdot \frac{e V_{y}}{E},
$$

where $R_{34}(=10 \mathrm{~m})$ is the optics coefficient that converts a beam vertical angle into a beam vertical displacement,

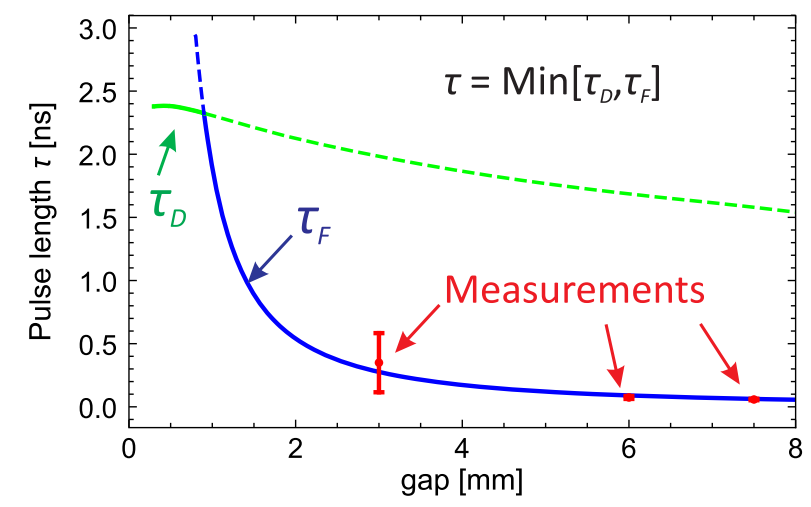

FIG. 11. Plot of simulated filling time (blue), simulated decay time (green) and of the pulse length measured with interferometer (red) as a function of the gap. When considering the surface wave, in the large gap regime, the pulse length is dominated by the filling time of the structure. given by the deflecting voltage $V_{y}$ and the beam energy $E$. For different beam-structure distances we measured the vertical deflection of the beam centroid $(\Delta y)$ on the BPM No. 3315, and the corresponding deflecting voltage was calculated using the electron beam optics parameter $R_{34}$. In this measurement, we assume that the $20.35 \mathrm{GeV}$ beam offset at the end of the structure due to the deflection is negligible and it only changes its vertical momentum.

We measured the deflecting voltages with the BPM located downstream of the vacuum chamber and calculated the correspondent kick factor. We compared the measurements with simulations performed with GdfidL [23] and with the computer code "NOVO" [24-27], considering a beam with $\sigma_{z}=25 \mu \mathrm{m}$, showing the kick factor plots in Fig. 12. The computer code "NOVO" simulates geometries with a constant cross-section. The NOVO geometry that approximates the FACET 3D structure, is shown in Fig. 13. The top view of the regular cell (in Fig. 13) has the same cross section of the regular cell used in our experiment (shown in Fig. 4 of paper [1]).

In Fig. 12 the measured deflections are systematically lower than the simulations. The systematic deviation is about $30 \mu \mathrm{m}$. Possible sources of deviations are unknown 


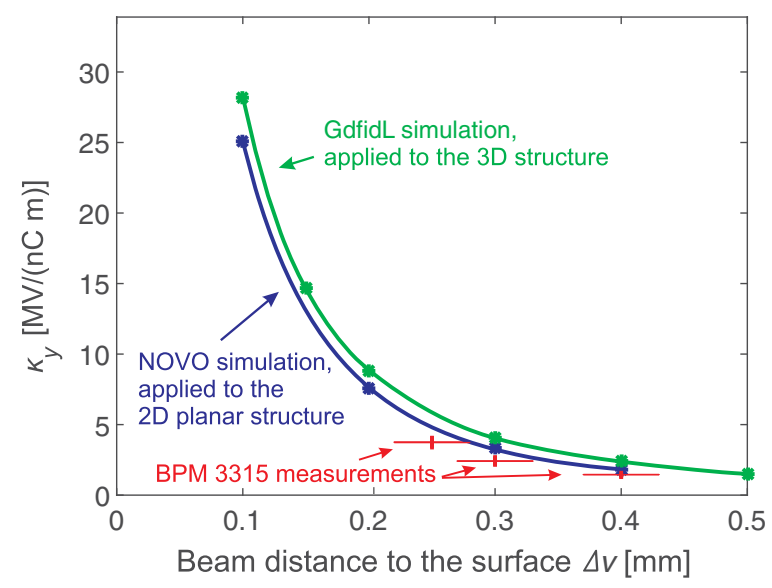

FIG. 12. Plot of the kick factor of the deflection towards the structure, measured with the BPM downstream of the vacuum chamber, with a beam with $\sigma_{z}=25 \mu \mathrm{m}$. Comparison with NOVO simulation (simulating the 2D, planar structure of Fig. 13) and with GdfidL (simulating the 3D structure shown on Fig. 5). Simulation performed with a beam with $\sigma_{z}=25 \mu \mathrm{m}$.

transverse and longitudinal shape of the beam and optics inaccuracies. The transverse size of the beam which is $\sigma_{y}=$ $30 \mu \mathrm{m}$ is consistent with the size of the systematic error. The beam deflection is calculated from the $R_{34}$ coefficient which is determined with finite tolerances from beam optics parameters which may introduce additional errors.

Our studies suggest possible application of the surface wave for beam manipulations, such as beam dechirpers [2832], or beam diagnostic, such as passive deflectors [33,34]. The deflecting wakefields create a ramp of deflecting forces along the length of the bunch, which could be used for bunch measurements at sub-fs resolution [28,33,34].

We compared our data with the results from the experiments with the SLAC dechirper [28-32]. With the dechirper the bunch charge was $0.1-0.2 \mathrm{nC}$ and the structure length was $2 \mathrm{~m}$; in our FACET experiment the bunch charge was 2.7$3.2 \mathrm{nC}$ on and the structure length was $0.1 \mathrm{~m}$ (see Table III). In

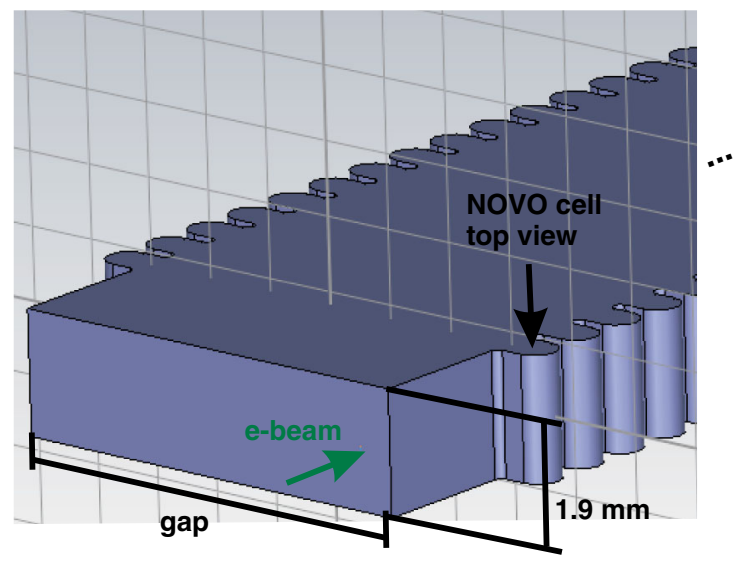

FIG. 13. Planar geometry with rectangular cross-section, simulated with the code NOVO, that approximates the FACET 3D structure. our FACET experiment we measured deflecting voltages comparable to ones achieved with the dechirper. The product charge times length are similar for both experiments, therefore the kick factor has the same order of magnitude. Experiments with the SLAC dechirper show potential for sub-fs resolution [28-32], therefore with our setup and FACET beam parameters we expect to see similar resolution.

\section{E. RF breakdown rate measurement}

The breakdown rate measurement was performed with an instrument we invented for open structures which we call the "arc-detector." The two metal halves that compose the structure were electrically insulated from ground and each other using plastic film. We connected the two metal halves to an oscilloscope (this provides the "current monitor signal"). Since breakdowns generate electron and ion currents, a signature of an $\mathrm{rf}$ breakdown is a spike in the acquired current monitor signal. We assumed that these voltage spikes are due to currents generated by rf breakdowns.

We defined a constant threshold for the current monitor signal, chosen to be higher than the electromagnetic ambient noise and field emission currents. Spikes with larger values than this threshold are tagged as rf breakdowns. The breakdown rate is the ratio of the breakdown events over the total number of pulses.

During the breakdown rate measurement the beam is horizontally centered above the structure cells. We changed the gradient by varying the beam-structure " $\Delta v$ " distance of Fig. 5. The results are presented in Fig. 14, for the shift of Nov 30, 2015, with $\sigma_{z}=25 \mu \mathrm{m}$, and $q=2.7 \mathrm{nC}$. The gap was $7.5 \mathrm{~mm}$. The rf measured pulse length was $60 \mathrm{ps}$. The peak electric field was calculated with HFSS. Unlike in the previous experiments, the current monitor signal was not anymore clear. The dark current was very complex and always correlated with the field. For measuring the

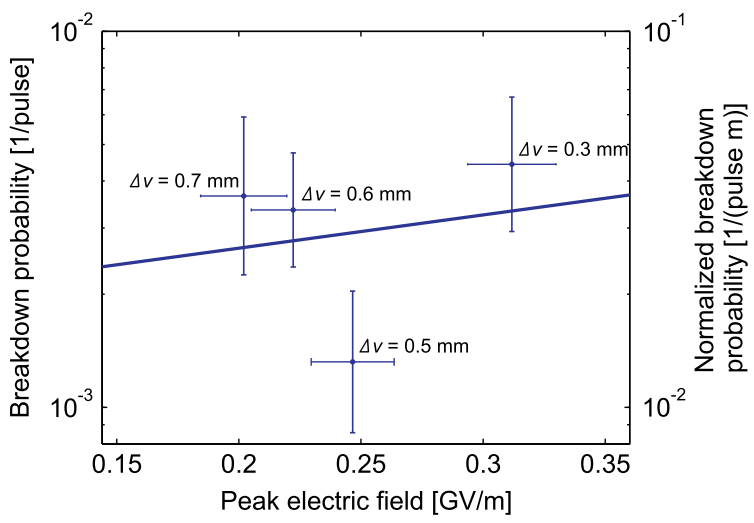

FIG. 14. Breakdown rate measurement of the surface wave, performed in the shift of Nov 30, 2015, with $\sigma_{z}=25 \mu \mathrm{m}$, and $q=2.7 \mathrm{nC} ; \Delta v$ is the beam-structure distance. The gap was $7.5 \mathrm{~mm}$, the rf pulse length was $60 \mathrm{ps}$. The peak electric field of the surface wave synchronous with the ultrarelativistic electron beam was calculated with HFSS. 
breakdown rate of Fig. 13, we used the following method: we setup a threshold for breakdown detection (see Fig. 18 of paper [3]), at a relatively high level, to prevent it from triggering on the dark current signal. We conjecture that this method allows us to detect obvious breakdowns.

In these experiments, the vacuum level was about $10^{-6}$ Torr. We conjecture that the breakdown rate is expected to improve with a better vacuum and more conditioning time. The presence of the FACET beam could have increased the breakdown rate. The beam halo was intercepted by the structure and, on a few occasions, the whole beam was dumped into the structure due to linac faults.

The surface wave breakdown rate shown in Fig. 14 is similar to that of the closed structure. In the surface wave experiments, for a gap $=7.5 \mathrm{~mm}$ and $\Delta v=0.3 \mathrm{~mm}$ we obtained a breakdown rate of $4 \times 10^{-3}$ per pulse at a peak electric field of $E_{\max }=0.3 \mathrm{GV} / \mathrm{m}$. In the closed gap experiment with the same structure, for a gap $=1 \mathrm{~mm}$ we obtained a breakdown rate of $3 \times 10^{-3}$ at a peak electric field of $E_{\max }=0.36 \mathrm{GV} / \mathrm{m}$ [4].

In Fig. 14 the measured breakdown rate does not show a very steep field dependence. Typical statistics measured with high gradient $\mathrm{X}$-band cavities show a very steep field dependence on the breakdown rate. This difference could be caused by the following physics: influence of the beam halo could have consistently interfered with the breakdown statistics and distorted the expected steep field dependence. This is because in the surface wave measurements the beam travels at sub-mm distances from the structure surface. Moreover, in our FACET experiments, we were not measuring the steady state breakdown rate, in which we expect to see the steep field dependence, since we were limited to a few shifts of few hours each, the saturated breakdown rate could not be achieved.

\section{CONCLUSIONS}

This paper explores the physics of rf breakdowns in mmwave open accelerating structures excited by an ultrarelativistic electron beam at the FACET facility at SLAC National Accelerator Laboratory. The structure gap is opened up to $7.5 \mathrm{~mm}$ or 2.4 times the wavelength of the mode synchronous with the electron beam. For all gap values, the rf power induced in the structure is guided by the corrugations with no radiative losses. This behavior is consistent with the so called "surface wave" and its properties were characterized. Measurements confirmed that with larger gap, wave parameters such as frequency and group velocity are less dependent on gap width. This beam-wave interaction was characterized by several methods: by measuring the beam deflection towards a structurehalf by using beam position monitors and beam profile monitors, by measuring the radiated rf energy with a pyroelectric detector, and by measuring the frequency and rf pulse length with a mm-wave interferometer. At the largest gap of $7.5 \mathrm{~mm}$, the parameters of the surface wave are as follows: the frequency is $95 \mathrm{GHz}$, the pulse length is $60 \mathrm{ps}$, and the group velocity is $0.84 \mathrm{c}$.

The beam distance from the surface of a structure-half was varied to modulate the surface wave amplitude and the rf breakdown rate was then measured. This is the first breakdown rate measurement for a surface wave excited in a mm-wave accelerating structure. With a gap of $7.5 \mathrm{~mm}$ and a beam distance of $0.3 \mathrm{~mm}$ from the surface, the breakdown rate was $4 \times 10^{-3}$ per pulse with a peak surface electric field of $0.3 \mathrm{GV} / \mathrm{m}$ and a pulse length of $60 \mathrm{ps}$.

The surface wave is a potential tool for beam manipulations, for example as a beam dechirper [28-32], or for beam diagnostics such as a passive rf deflector $[33,34]$. The deflecting wakefields create a ramped deflecting force along the length of the bunch which could be used for bunch measurements with sub-fs resolution $[28,33,34]$.

Our structure has input and output rf power couplers and therefore can be driven by an if source. To enable such applications, we are developing methods for coupling MW level power into mm-wave accelerating structures [35,36]. Understanding the properties of $\mathrm{mm}$-wave structures with high accelerating and deflecting gradients may open paths for novel methods of commissioning and operation of free electron lasers and compact synchrotron light sources.

\section{ACKNOWLEDGMENTS}

Work supported by the U.S. DOE under Contract No. DE-AC02-76SF00515. We thank all the E204 experiment crew: Juan Cruz Jr., RF Diagnostics: Spencer Gessner, Oliver Williams (UCLA), Filippos Toufexis. SEM: Chris Pearson. Machine optics and other informations: Erik Adli, Carl Andreas Lindstrom, Michael Litos. We thank Vitaly Yakimenko and FACET team for excellent support. We thank Warner Bruns for his support with the GdfidL calculation software.

[1] M. Dal Forno, V. Dolgashev, G. Bowden, C. Clarke, M. Hogan, D. McCormick, A. Novokhatski, B. Spataro, S. Weathersby, and S. G. Tantawi, rf breakdown tests of mmwave metallic accelerating structures, Phys. Rev. Accel. Beams 19, 011301 (2016).

[2] M. Dal Forno, V. Dolgashev, G. Bowden, C. Clarke, M. Hogan, D. McCormick, A. Novokhatski, B. Spataro, S. Weathersby, and S. G. Tantawi, Experimental measurements of rf breakdowns and deflecting gradients in mmwave metallic accelerating structures, Phys. Rev. Accel. Beams 19, 051302 (2016).

[3] M. Dal Forno, V. Dolgashev, G. Bowden, C. Clarke, M. Hogan, D. McCormick, A. Novokhatski, B. O'Shea, B. Spataro, S. Weathersby, and S. G. Tantawi, rf breakdown measurements in electron beam driven $200 \mathrm{GHz}$ copper and copper-silver accelerating structures, Phys. Rev. Accel. Beams 19, 111301 (2016).

[4] M. D. Forno, V. Dolgashev, G. Bowden, C. Clarke, M. Hogan, D. McCormick, A. Novokhatski, B. O'Shea, B. 
Spataro, S. Weathersby, and S. G. Tantawi, High gradient tests of metallic mm-wave accelerating structures, Nucl. Instrum. Methods Phys. Res., Sect. A 864, 12 (2017).

[5] Facility for advanced accelerator experimental tests, (FACET), http://facet.slac.stanford.edu/.

[6] D. W. Dupen, H. A. Hogg, and G. A. Loew, The Stanford Two Mile Accelerator, edited by R. B. Neal (W. A. Benjamin, Inc., New York, 1968), p. 1169, http://www .slac.stanford.edu/library/2MileAccelerator/2mile.htm; Report No. SLAC-REPRINT-1968-001.

[7] C. C. Cutler, Genesis of the corrugated electromagnetic surface, in Proceedings of IEEE Antennas and Propagation Society International Symposium and URSI National Radio Science Meeting (IEEE, Seattle, WA, 1994), https:// doi.org/10.1109/APS.1994.408225.

[8] G. Goubau, Single-conductor surface-wave transmission lines, Proc. IRE 39, 619 (1951).

[9] G. Goubau, On the excitation of surface waves, Proc. IRE 40, 865 (1952).

[10] G. Goubau, Open wire lines, IRE Trans. Microwave Theory Tech. 4, 197 (1956).

[11] W. Rotman, A study of single-surface corrugated guides, Proc. IRE 39, 952 (1951).

[12] G. John, Electromagnetic surface waveguides-A review, IEE-IERE Proceedings, India 15, 139 (1977).

[13] H. M. Barlow, Surface waves, Proc. IRE 46, 1413 (1958).

[14] S. J. Smith and E. M. Purcell, Visible light from localized surface charges moving across a grating, Phys. Rev. 92, 1069 (1953).

[15] J. H. Booske, R. J. Dobbs, C. D. Joye, C. L. Kory, G. R. Neil, G. S. Park, J. Park, and R. J. Temkin, Vacuum electronic high power terahertz sources, IEEE Trans. Terahertz Sci. Technol. 1, 54 (2011).

[16] High frequency structural simulator, (HFSS), http://www .ansys.com/Products/.

[17] V. Dolgashev, S. Tantawi, Y. Higashi, and B. Spataro, Geometric dependence of radio-frequency breakdown in normal conducting accelerating structures, Appl. Phys. Lett. 97, 171501 (2010).

[18] Edm Department Inc. (Bartlett, IL, USA), http://www .edmdept.com/.

[19] J. Wang, J. Lewandowski, J. Van Pelt, C. Yoneda, B. Gudkov, G. Riddone, T. Higo, and T. Takatomi, Fabrication technologies of the high gradient accelerator structures at $100 \mathrm{MV} / \mathrm{m}$ range, Proceedings of IPAC 2010, May 2010, Kyoto, Japan, THPEA064 (2010), https://accelconf .web.cern.ch/accelconf/IPAC10/papers/thpea064.pdf.

[20] J. Wang, Surface treatment issues for RF structure fabrication, in X-band Accelerating Structure Review (CERN, Geneva, Switzerland, 2014), https://indico.cern.ch/event/ 346988/contributions/1746821/attachments/684956/ 940866/Juwen_Wang_CERN__Structure_Review_2014 .pdf.

[21] V. A. Dolgashev, G. Bowden, Y. Ding, P. Emma, P. Krejcik, J. Lewandowski, C. Limborg, M. Litos, J. Wang, and D. Xiang, Design and application of multimegawatt X-band deflectors for femtosecond electron beam diagnostics, Phys. Rev. ST Accel. Beams 17, 102801 (2014).

[22] P. Craievich, M. Petronio, S. G. Biedron, D. Castronovo, M. D. Forno, S. D. Mitri, N. Faure, D. L. Civita, G. Penco,
L. Rumiz, L. Sturari, R. Vescovo, and D. Wang, Implementation of radio-frequency deflecting devices for comprehensive high-energy electron beam diagnosis, IEEE Trans. Nucl. Sci. 62, 210 (2015).

[23] Gdfidl, (Germany), http://www.gdfidl.de/.

[24] A. Novokhatsky, The computer code NOVO for the calculation of wake potentials of the very short ultrarelativistic bunches, Report No. SLAC-PUB-11556, 2005, http://www.slac.stanford.edu/cgi-wrap/getdoc/slac-pub11556.pdf.

[25] A. Novokhatski, Field dynamics of coherent synchrotron radiation using a direct numerical solution of Maxwell's equations, Phys. Rev. ST Accel. Beams 14, 060707 (2011).

[26] A. Novokhatsky, Modeling of coherent synchrotron radiation using a direct numerical solution of Maxwell's equations, SLAC Report No. SLAC-PUB-15258, 2012, http://slac.stanford.edu/pubs/slacpubs/15250/slac-pub15258.pdf.

[27] V. Balakin, I. Koop, A. Novokhatski, A. S. Skrinski, and V. Smirnov, Beam dynamics of a colliding linear electronpositron beam (vlepp), BINP Report No. SLAC-TRANS0188, 1978, http://slac.stanford.edu/pubs/slactrans/ trans01/slac-trans-0188.pdf.

[28] A. Novokhatski, Wakefield potentials of corrugated structures, Phys. Rev. ST Accel. Beams 18, 104402 (2015).

[29] M. Guetg et al., Commissioning of the RadiaBeam/SLAC dechirper, in Proceedings, of the 7th International Particle Accelerator Conference (IPAC 2016): Busan, Korea, 2016 (2016), p. MOPOW044, http://accelconf.web.cern.ch/ accelconf/ipac2016/papers/mopow044.pdf.

[30] M. Dal Forno et al., Electron beam excitation of a surface wave in mm-wave open accelerating structures, in Proceedings of the 7th International Particle Accelerator Conference (IPAC 2016): Busan, Korea, 2016 (2016), p. MOPMW040, http://accelconf.web.cern.ch/accelconf/ ipac2016/papers/mopmw040.pdf.

[31] A. Novokhatski et al., RadiaBeam/SLAC dechirper as a passive deflector, in Proceedings of the 7th International Particle Accelerator Conference (IPAC 2016): Busan, Korea, 2016 (2016), p. MOPOW046, http://accelconf .web.cern.ch/accelconf/ipac2016/papers/mopow046.pdf.

[32] J. Zemella, K. Bane, A. Fisher, M. Guetg, Z. Huang, R. Iverson, P. Krejcik, A. Lutman, T. Maxwell, A. Novokhatski, G. Stupakov, Z. Zhang, M. Harrison, and M. Ruelas, Measurements of wake-induced electron beam deflection in a dechirper at the Linac Coherent Light Source, Phys. Rev. Accel. Beams 20, 104403 (2017).

[33] S. Bettoni, P. Craievich, A. A. Lutman, and M. Pedrozzi, Temporal profile measurements of relativistic electron bunch based on wakefield generation, Phys. Rev. Accel. Beams 19, 021304 (2016).

[34] V. Dolgashev, Attosecond diagnostics of muti-GeV electron beams using W-band deflectors, in Proceeding of FEIS-2: Femtosecond Electron Imaging and Spectroscopy, East Lansing, Michigan (2015), http://bt.pa.msu.edu/FEIS2/talks/FEIS-2_Dolgashev.pdf.

[35] E. A. Nanni, V. A. Dolgashev, A. Haase, J. Neilson, S. Tantawi, S. C. Schaub, R. J. Temkin, and B. Spataro, Prototyping high-gradient $\mathrm{mm}$-wave accelerating structures, J. Phys. Conf. Ser. 874, 012039 (2017). 
[36] E. Nanni, M. Dal Forno, V. Dolgashev, J. Neilson, S. Schaub, S. Tantawi, and R. Temkin, mm-wave standingwave accelerating structures for high-gradient tests, in Proceedings of the 7th International Particle Accelerator
Conference (IPAC 2016): Busan, Korea, 2016 (2016), p. THPOR044, http://accelconf.web.cern.ch/accelconf/ ipac2016/papers/thpor044.pdf. 